\title{
HYPOCOMPLEMENTEMIC VASCULITIS ASSOCIATED WITH COVID 19 VACCINE: CASE REPORT
}

\author{
Rodrigo Lorenzetti Serrano ${ }^{1}$ \\ 1.Universidade Federal de São Paulo, São Paulo (SP), Brazil. \\ *Corresponding author: rodrigo.serrano@unifesp.br
}

\section{BACKGROUND}

With the advance of vaccination against COVID-19, the description of side effects also grows. The most frequent side effects are: pain at the application site, nausea, myalgia, fever and nausea. Among the less described effects are the appearance of purpuric lesions, but the neurological involvement has an unknown frequency. In this case report, we present possible rare side effects in a patient without any past medical history.

\section{CASE REPORT}

A 44-year-old woman, smoker and with no other comorbidities, was admitted to emergency care due to knee arthralgia associated with nonpruritic purpuric lesions in the lower limbs associated with sudden numbness in her legs and gait impairment in the last 2 days. She denied recent infections or travels, and was vaccinated against COVID-19 (AstraZeneca) 9 days ago. On neurological examination, a symmetrical reduced proximal and more important distal muscle strength in the lower limbs was noted. She also had tactile, thermal and vibratory hypoesthesia distal to the knees bilaterally. Cerebrospinal fluid puncture was performed without inflammatory characteristics or protein-cytological dissociation, neuroaxis magnetic resonance imaging without signal alteration and electroneuromyography with $\mathrm{F}$ wave latency. Laboratory findings: red blood count with mild anemia ( $\mathrm{Hb} 11.8 \mathrm{mg} / \mathrm{dL})$, normal renal function and 24-h proteinuria, hypocomplementemia $(\mathrm{CH} 100=42$ and $\mathrm{C} 2=55)$, negative rheumatoid factor, ANA, ANCA. Biopsy of skin lesions showed lymphocytic vasculitis. We prescribed pulse therapy with dexamethasone $160 \mathrm{mg}$ for 3 days (due to the unavailability of methylprednisolone) and cyclophosphamide $0.73 \mathrm{~g} / \mathrm{m}^{2}$. During hospitalization patient showed improvement in, muscle strength and complete resolution of hypoesthesia and purpuric lesions in lower limbs. Hospital discharge after 12 days with prednisone $1 \mathrm{mg} / \mathrm{kg} / \mathrm{day}$ and new scheduled cyclophosphamide infusions.

\section{CONCLUSION}

This case shows a previously healthy woman with neurologic and cutaneous manifestations compatible with vasculitis. With the increase of vaccinated people, the epidemiologic surveillance is fundamental to monitor possible rare side effects associated with vaccination.

\section{KEYWORDS}

Vasculitis, COVID-19, Vaccine. 